\title{
Época de coleta das estacas, do uso de fitorregulador de enraizamento e de diferentes tipos de enxertos na produção de mudas de figueira 'Roxo de Valinhos'
}

\author{
Time of cutting collection, use the rooting fitorregulador and \\ different types of grafts in the seedlings production of ' $R$ oxo de \\ Valinhos' fig tree
}

\author{
Tailene Elisa Kotzi ; Rafael Pio ${ }^{2 *}$; Edvan Alves Chagas 3 ; \\ Marcelo Angelo Campagnolo ${ }^{4}$; José Emílio Bettiol Neto5; Maraisa Hellen Tadeu ${ }^{6}$
}

Resumo

O objetivo deste trabalho foi verificar a viabilidade de enxertia de mesa na figueira 'Roxo de Valinhos', por borbulhia. No primeiro experimento, estacas lenhosas da porção mediana dos ramos, com $20 \mathrm{~cm}$ de comprimento, foram coletadas nos meses de maio, junho, julho, agosto e setembro. Foram realizadas enxertias de mesa pelo processo de borbulhia tipo placa (retirada de $10 \mathrm{~mm}^{2}$ de casca no entrenó da estaca, inserindo-se uma borbulha extraída com mesma dimensão das gemas verdes e inchada da porção apical do ramo). No segundo experimento, as estacas foram coletadas em julho, sendo realizadas enxertias de mesa pelo processo de borbulhia, por diferentes tipos: placa, " $\mathrm{T}$ " normal, " $\mathrm{T}$ " invertido e janela. Após a inserção da borbulha, amarrou-se com fita plástica, durante 30 dias, deixando a gema exposta. Metade das estacas de cada experimento teve suas bases tratadas em $2000 \mathrm{mg} \mathrm{L}^{-1}$ de AIB por 10 segundos e a outra metade permaneceu sem tratamento. As estacas foram enterradas em areia, sob telado (50\% de luminosidade). Aos 120 dias após a enxertia, foi mensurada a porcentagem total de borbulhas vivas, brotadas, borbulhas vivas em porta-enxertos enraizados e o comprimento médio da brotação. A melhor época para a realização da enxertia é entre os meses de junho a agosto; a enxertia por borbulhia deve ser realizada pelo tipo "T" normal; as estacas enxertadas devem ser tratadas com AIB. Palavras-chave: Ficus carica L, multiplicação, borbulhia, estaquia e ácido indolbutírico

\footnotetext{
${ }^{1}$ Acadêmica em Agronomia da Universidade Estadual do Oeste do Paraná, UNIOESTE, Rua Pernambuco, 1.777, Caixa Postal 1.008, Centro, 85.960-000, Marechal Cândido Rondon, PR. E-mail: taileneelisa@hotmail.com

2 Prof. Adjunto, Universidade Federal de Lavras, UFLA, Departamento de Agricultura, Caixa Postal 3037, 37200-000, Lavras, MG. Bolsista Produtividade em Pesquisa CNPq. E-mail: rafaelpio@hotmail.com

${ }^{3}$ Pesquisador Científico da Empresa Brasileira de Pesquisa Agropecuária, EMBRAPA CPAFRR, Rodovia 174, Km 8, Caixa Postal 133, 69301-970, Boa Vista, RR. Bolsista Produtividade em Pesquisa CNPq. E-mail: echagas@cpafrr.embrapa.br

${ }^{4}$ Pós-graduando do curso de Doutorado em Agronomia, Universidade Estadual do Oeste do Paraná, UNIOESTE, Rua Pernambuco, 1.777, Caixa Postal 1.008, Centro, 85.960-000, Marechal Cândido Rondon, PR. Bolsista do CNPq. E-mail: campa_bio@yahoo. com.br

5 Pesquisador Científico do Centro Avançado de Pesquisa Tecnológica do Agronegócio de Frutas, Instituto Agronômico, IAC, Av. Luiz Pereira dos Santos, 1500, Corrupira, 13214-820, Jundiaí, SP. E-mail: bettiolneto@iac.sp.gov.br

${ }^{6}$ Graduanda em Agronomia, Universidade Federal de Lavras, UFLA, Caixa Postal 3037, 37200-000, Lavras, MG. Bolsista de Iniciação Científica do CNPq. E-mail: maraisaht@yahoo.com.br

* Autor para correspondência
} 


\begin{abstract}
This work aimed to develop a protocol of table grafting in 'Roxo de Valinhos' fig tree by bud method. The first experiment, woody cutting of the medium portion, with $20 \mathrm{~cm}$ of length, were collected of May, June, July, August and September. Table grafting was accomplished by bud method plate type (it cuts of $10 \mathrm{~mm}^{2}$ among two buds of the cutting, bud interfering of same dimension, extracted of the green and swollen buds of the cutting apical portion). In the second experiment, the cuttings were collected in July, being accomplished table grafting by the bud methods, for different types: plate, normal " $T$ ", inverted " $\mathrm{T}$ " and window. After the insert of the bud, in two experiments, they were tied the bud with plastic ribbon, leaving the exposed of the bud. Half of the grafted cutting had its bases negotiated in $2000 \mathrm{mg}$ $\mathrm{L}^{-1}$ of IBA by 10 seconds and the other half stayed without treatment. The cuttings were buried in sand, under nursery with $50 \%$ of brightness. The 120 days after the grafting, the percentage of alive, sprouting buds, alive buds in taken root rootstocks and the medium length of the sprouting were evaluated. The table grafting is among of June to August, by normal " $T$ " and the cutting treatment with IBA.
\end{abstract}

Key words: Ficus carica L, multiplication, cutting and indolbutyric acid

\section{Introdução}

A figueira (Ficus carica L.) constituiu-se uma das mais importantes frutíferas de clima temperado cultivada, devido a sua larga adaptação climática, rusticidade e fins de aproveitamento de seus subprodutos, não incipiente a utilização nobre de seus frutos, mas também no aproveitamento de suas folhas na fabricação de bebidas fermentadas e ramos como propágulos (ALVARENGA et al., 2007).

O Brasil é o maior produtor de figos do hemisfério Sul, ocupando a $14^{\mathrm{a}}$ posição mundial (FAO, 2008). São Paulo é o único estado brasileiro exportador e, o maior produtor de figos voltados para a produção de fruta fresca. Porém, analisando a área de cultivo no Estado, nota-se que houve acentuado decréscimo nos últimos anos, com redução da área cultivada em aproximadamente 21\%, nos últimos 12 anos, passando de 487 ha em 1994, para 386 ha em 2006 (PIO et al., 2007; IBGE, 2008). Já no Estado do Paraná, a ficicultura encontra-se incipiente, com 179 ha em cultivo, no entanto, com grande possibilidade de expansão.

Além da elevada exploração imobiliária ocorrida no principal pólo ficícola de São Paulo, a região do Circuito das Frutas da grande Campinas, a hegemonia do único cultivar explorado comercialmente ('Roxo de Valinhos'), vem proporcionando sérios problemas aos ficicultores paulistas. Isso deve-se ao fato da cultivar apresentar sérios problemas de ordem fitossanitária, como os nematóides (Meloidogyne incógnita e Heterodera fici) e a seca-da-mangueira (Ceratocystis fimbriata Ell. \& Halst.), enfermidades de solo que vem a cada ano ocasionando prejuízo nos pomares do Estado (RIBEIRO, 1999). Assim, utilização de porta-enxertos resistentes seria a única alternativa para o cultivo de figueiras em locais infestados.

Na década de 60, o Instituto Agronômico (IAC) detinha aproximadamente 25 cultivares de $F$. carica (RIGITANO, 1955), porém, esses cultivares foram se perdendo frente ao baixo interesse pelos ficicultores em diversificar o 'Roxo de Valinhos'.

Quanto a outras espécies de figueira, Condit (1950) cita que o F. glomerata possui imunidade satisfatória em relação ao ataque de nematóides. Tyler (1944) relatou que as espécies $F$. cocculifolia, F. pumila e F. racemosa são consideradas tolerantes ao ataque dos nematóides causadores de galha nas raízes. Davies, Lazarte e Joiner (1982) verificaram que a espécie $F$. pumila possui alto potencial rizogênico na propagação por estacas lenhosas, o que não é um empecilho na maioria das espécies do gênero Ficus (POOLE; CONOVER, 1984).

Considerando a introdução de outros cultivares e espécies do gênero Ficus, a quantidade de propágulos vegetativos advindo de outros países é reduzida, o que onera os programas de melhoramento, frente a demanda de longo prazo para a multiplicação dos 
cultivares. A estaquia é a técnica mais difundida na multiplicação da figueira, no entanto, os índices de enraizamento dependem de uma série de fatores, dentre eles, a época de coleta das estacas, a posição do ramo a ser coletada, o tratamento com fitoreguladores e o cultivar ou espécie, podendo tais índices variar de 10\% a 80\% (PIO et al., 2006).

É necessário, previamente à introdução de cultivares ou espécies do gênero Ficus tolerantes aos nematóides, testar o potencial propagativo das demais espécies resistentes encontradas no Brasil, verificar a viabilidade e o domínio da técnica de enxertia. Mesmo tratando-se da enxertia intraespecífica (mesma espécie) e intra-varietal, o domínio da técnica contribuiria na otimização do tempo necessário para a multiplicação e nos testes à campo.

Nesse contexto, o presente trabalho teve como objetivo verificar a influência da época de coleta das estacas, do uso de fitorregulador de enraizamento e de diferentes tipos de enxertos na produção de mudas de figueira 'Roxo de Valinhos'.

\section{Material e Métodos}

Foram desenvolvidos dois experimentos, entre os meses de maio de 2007 a janeiro de 2008, na Universidade Estadual do Oeste do Paraná (UNIOESTE), Marechal Cândido Rondon-PR. Os propágulos utilizados em ambos os experimentos foram coletados em um pomar comercial de quatro anos de idade, de figueira 'Roxo de Valinhos', situado no município de Quatro Pontes-PR.

As estacas lenhosas, coletadas da porção mediana dos ramos, foram padronizadas com $20 \mathrm{~cm}$ de comprimento, $15 \mathrm{~mm}$ de diâmetro e quatro nós, sendo efetuado um corte em bisel na base da estaca e outro reto no ápice. Após a coleta, as estacas foram mantidas por um dia entre camadas de jornal umedecido, para facilitar a operação de enxertia.

No primeiro experimento, foram coletadas estacas na primeira semana dos meses de maio, junho, julho, agosto e setembro. Foram realizadas enxertias de mesa pelo processo de borbulhia tipo placa. Fez-se cortes retirando-se $10 \mathrm{~mm}^{2}$ de casca entre dois nós da estaca, inserindo-se nesse local uma borbulha extraída com as mesmas dimensões, das gemas verdes e inchadas da porção apical do ramo, coletadas nos referidos meses.

No segundo experimento, as estacas foram coletadas no final do mês de julho, adotando-se os mesmos procedimentos descritos no primeiro experimento. Foram realizadas enxertias de mesa pelo processo de borbulhia, em diferentes tipos: placa (cortes quadrados de $10 \mathrm{~mm}^{2}$ ), "T" normal e " $\mathrm{T}$ " invertido (corte superficial na casca da estaca, de $20 \mathrm{~mm}$ na vertical e $10 \mathrm{~mm}$ na horizontal) e janela aberta (dois cortes paralelos na horizontal de $10 \mathrm{~mm}$ e um na vertical de $20 \mathrm{~mm}$ ).

Após a inserção da borbulha, em ambos os experimentos, foi realizado o amarrio das borbulhas com fita plástica deixando a gema exposta. Metade da quantia das estacas de cada experimento teve suas bases tratadas em $2000 \mathrm{mg} \mathrm{L}^{-1}$ de AIB por 10 segundos (PIO et al., 2006) e a outra metade permaneceu sem tratamento. Posteriormente, as estacas foram enterradas à $2 / 3$ de seu comprimento em leito de areia, sob telado (50\% de luminosidade), umedecido diariamente.

O delineamento utilizado foi o inteiramente casualizado, no primeiro experimento adotouse o esquema fatorial $5 \times 2$ (época de realização da enxertia e tratamento com AIB) e no segundo experimento o esquema fatorial $4 \times 2$ (tipos de enxertia e tratamento com AIB). Em ambos os experimentos, utilizaram-se quatro repetições e 10 enxertos por unidade experimental.

Aos 30 dias após a enxertia retirou-se o amarrio e aos 120 dias foi mensurada a porcentagem total de borbulhas vivas, porcentagem de borbulhas brotadas, porcentagem de borbulhas vivas em portaenxertos enraizados (borbulhas vivas em estacas enraizadas) e o comprimento médio da brotação.

Os dados foram submetidos à análise de variância 
pelo teste $\mathrm{F}$ e as médias comparadas pelo teste de Tukey. As análises foram realizadas pelo programa computacional Sistema para Análise de Variância SISVAR.

\section{Resultados e Discussão}

No primeiro experimento, houve diferença significativa entre as épocas de enxertia para a porcentagem total de borbulhas vivas (PTBV) e borbulhas vivas em porta-enxertos enraizados (PBVPE) (Tabela 1). Houve interação significativa para a porcentagem de borbulhas brotadas e para o comprimento médio das brotações entre os fatores em estudo.

Para a porcentagem de borbulhas vivas, enxertias realizadas entre os meses de junho a agosto promoveram os melhores resultados, registrandose mais de $78 \%$ de sobrevivência (Tabela 1). Em relação à porcentagem de borbulhas vivas em portaenxertos enraizados, enxertias realizadas entre os meses de maio a agosto não diferiram entre si, obtendo-se índices superiores a 91\% (Tabela 1).

Tabela 1. Porcentagem total de borbulhas vivas (PTBV), borbulhas vivas em porta-enxertos enraizados (PBVPE), borbulhas brotadas (PBB) e comprimento médio da brotação (CMB) de figueira 'Roxo de Valinhos' (Ficus carica L.), obtida por enxertia de mesa em estacas lisas medianas, tratadas ou não com AIB (2000 mg L L-1), em diferentes épocas. Marechal Cândido Rondon-PR, UNIOESTE, 2008.

\begin{tabular}{|c|c|c|c|c|c|c|}
\hline \multirow{2}{*}{$\begin{array}{c}\text { Época } \\
\text { da enxertia }\end{array}$} & \multirow[t]{2}{*}{ PTBV (\%)* } & \multirow[t]{2}{*}{ PBVPE (\%) } & \multicolumn{2}{|c|}{ PBB (\%) } & \multicolumn{2}{|c|}{ CMB (mm) } \\
\hline & & & $\begin{array}{l}\text { Sem } \\
\text { AIB }\end{array}$ & $\begin{array}{l}\text { Com } \\
\text { AIB }\end{array}$ & $\begin{array}{l}\text { Sem } \\
\text { AIB }\end{array}$ & $\begin{array}{l}\text { Com } \\
\text { AIB }\end{array}$ \\
\hline Maio & 54,6 b & $93,7 \mathrm{a}$ & $0 \mathrm{Aa}$ & $5,0 \mathrm{Ab}$ & 0 Ac & $7,4 \mathrm{Ab}$ \\
\hline Jun. & $87,2 \mathrm{a}$ & 96,6 a & $5,0 \mathrm{Ba}$ & $17,5 \mathrm{Aa}$ & $31,7 \mathrm{Aa}$ & $31,7 \mathrm{Aa}$ \\
\hline Jul. & $90,5 \mathrm{a}$ & $100,0 \mathrm{a}$ & $0 \mathrm{Ba}$ & $15,0 \mathrm{Aa}$ & $0 \quad \mathrm{Bc}$ & 25,4 Aa \\
\hline Ago. & $78,2 \mathrm{a}$ & $91,4 \mathrm{a}$ & $2,5 \mathrm{Ba}$ & $20,5 \mathrm{Aa}$ & $17,7 \mathrm{Bb}$ & $30,1 \mathrm{Aa}$ \\
\hline Set. & $18,4 \mathrm{c}$ & $7,5 \mathrm{~b}$ & $0 \mathrm{Aa}$ & $3,4 \mathrm{Ab}$ & $0 \mathrm{Bc}$ & $11,5 \mathrm{Ab}$ \\
\hline C.V. $(\%)$ & 31,9 & 22,5 & & 12,9 & & 9,8 \\
\hline
\end{tabular}

* Médias seguidas pela mesma letra em maiúsculo na linha e mesma letra em minúsculo na coluna, não diferem entre si pelo teste de Tukey $(P<0,05)$.

Verifica-se a dependência entre a sobrevivência da borbulha e o enraizamento da estaca, pois, as borbulhas que permaneceram vivas nesse período, estavam enxertadas em porta-enxertos que enraizaram. Esse é um fator fundamental para o sucesso da enxertia de mesa em figueira. Notase que enxertias realizadas no mês de setembro não apresentaram bons resultados, devido às plantas já terem saído da dormência hibernal e conseqüentemente, ocorrer decréscimo no potencial rizogênico da estaca, influenciando diretamente na sobrevivência da borbulha (Tabela 1). Segundo Norberto et al. (2001), estacas medianas de figueira 'Roxo de Valinhos' apresentam alta porcentagem de enraizamento, quando coletadas em plantas entre o período de entrada da dormência (abril) até a época de realização da poda (julho); no entanto, coletas tardias depararam com acentuada queda do potencial rizogênico das estacas.

As estacas que não foram tratadas com o AIB apresentaram baixo percentual de brotação, independente do mês em que se realizou a enxertia. Porém, as que receberam aplicação do fitoregulador, apresentaram melhor índice de brotação em nas enxertias realizadas em agosto (20,5\%), que não diferiu dos meses de junho $(17,5 \%)$ e julho (15\%) (Tabela 1). Para o comprimento médio da brotação, os melhores resultados foram verificados nas enxertias realizadas entre os meses de junho e agosto, em estacas tratadas com o AIB (Tabela 1). 
Segundo Dutra e Kersten (1996), a influência da época de estaquia no enraizamento de estacas ocorre devido as variações no conteúdo dos cofatores na formação e no acúmulo de inibidores do enraizamento, o que provavelmente explique a resposta efetiva à aplicação do regulador ao longo das épocas testadas. No caso de estacas lenhosas de frutíferas de clima temperado, reservas mais abundante de carboidratos correlacionamse com maiores porcentagens de enraizamento e sobrevivência de estacas. Assim, a real importância dos carboidratos para formação de raízes é que a auxina requer fonte de carbono para a biossíntese de ácidos nucléicos e proteínas, levando à necessidade de energia e carbono para formação das raízes (HARTMANN et al., 2002).
No segundo experimento, houve interação significativa entre todas as variáveis mensuradas, à exceção da porcentagem de borbulhas vivas em porta-enxertos enraizados que não apresentou diferença significativa em função dos fatores em estudo.

A borbulhia tipo " $\mathrm{T}$ " normal destacou-se entre os demais, obtendo-se $90 \%$ de borbulhas vivas, independente do tratamento com o fitoregulador, não diferindo da enxertia tipo "T" invertido em estacas tratadas com AIB (Tabela 2). Em relação à porcentagem de borbulhas vivas em porta-enxertos enraizados, ocorreu mais de $97 \%$ de sobrevivência das borbulhas em estacas que enraizaram. Com esses resultados, reforça-se a necessidade do sucesso da rizogênese da estaca para ocorrer sucesso na enxertia de mesa.

Tabela 2. Porcentagem total de borbulhas vivas (PTBV), borbulhas vivas em porta-enxertos enraizados (PBVPE), borbulhas brotadas (PBB) e comprimento médio da brotação (CMB) de figueira 'Roxo de Valinhos' (Ficus carica L.), obtida por enxertia de mesa por diferentes metodologias, em estacas lisas medianas, tratadas ou não com AIB (2000 mg L ${ }^{-1}$ ). Marechal Cândido Rondon-PR, UNIOESTE, 2008.

\begin{tabular}{|c|c|c|c|c|c|c|c|}
\hline \multirow{3}{*}{$\begin{array}{l}\text { Metodologia } \\
\text { de enxertia }\end{array}$} & \multicolumn{2}{|c|}{ PTBV $(\%)^{*}$} & \multirow[t]{3}{*}{ PBVPE (\%) } & \multicolumn{2}{|c|}{ PBB (\%) } & \multicolumn{2}{|c|}{$\mathrm{CMB}(\mathrm{mm})$} \\
\hline & Sem & Com & & Sem & Com & Sem & Com \\
\hline & AIB & AIB & & AIB & AIB & AIB & AIB \\
\hline Placa & $72,5 \mathrm{Ab}$ & $77,5 \mathrm{Ab}$ & 100,0 ns & $\begin{array}{ll}0 \mathrm{Ba} \\
\end{array}$ & $15,0 \mathrm{Ab}$ & $\begin{array}{ll}0 & \mathrm{Bb}\end{array}$ & $25,4 \mathrm{Ab}$ \\
\hline T normal & $90,0 \mathrm{Aa}$ & $90,0 \mathrm{Aa}$ & 97,5 & $5,0 \mathrm{Ba}$ & $25,0 \mathrm{Aa}$ & $16,6 \mathrm{Ba}$ & $37,2 \mathrm{Aa}$ \\
\hline $\mathrm{T}$ invertido & $72,8 \mathrm{Bb}$ & $89,4 \mathrm{Aa}$ & 100,0 & 2,5 Aa & $2,5 \mathrm{Ac}$ & $10,6 \mathrm{Aa}$ & $13,4 \mathrm{Ac}$ \\
\hline Janela & $46,4 \mathrm{Bc}$ & $64,2 \mathrm{Ac}$ & 97,2 & $1,5 \mathrm{Aa}$ & $5,0 \mathrm{Ac}$ & $0 \mathrm{Bb}$ & $21,8 \mathrm{Ab}$ \\
\hline C.V. $(\%)$ & \multicolumn{2}{|c|}{21,5} & 5,0 & \multicolumn{2}{|c|}{31,5} & \multicolumn{2}{|c|}{16,4} \\
\hline
\end{tabular}

* Médias seguidas pela mesma letra em maiúsculo na linha e mesma letra em minúsculo na coluna, não diferem entre si pelo teste de Tukey $(P<0,05)$.

ns - não significativo, segundo o teste $\mathrm{F}$.

A enxertia por borbulhia tipo " $\mathrm{T}$ " normal em estacas tratadas com AIB, apresentou melhores resultados para a porcentagem de borbulhas brotadas $(25 \%)$ e para o comprimento médio da brotação (37,2 mm), em relação aos demais tipos de enxertia e também quando comparado à ausência do tratamento com AIB (Tabela 2). Essa ação secundária do AIB, em relação aos brotos dos enxertos, pode estar relacionada à melhoria expressiva na rizogênese, principalmente quanto ao aumento do número de raízes, o que ocasionou aumento na taxa respiratória da estaca e desencadeou a mobilização das reservas (carboidratos), favorecendo a emissão da brotação do enxerto.

O enraizamento de estacas é influenciado por substâncias hormonais localizadas endogenamente nas estacas. Dentre as auxinas sintéticas, o AIB é o fitoregulador mais comumente utilizado na indução do enraizamento adventício, por se tratar de uma 
substância fotoestável, de ação localizada e menos sensível à degradação biológica, em comparação às demais auxinas sintéticas (FACHINELLO; HOFFMANN; NACHTIGAL, 2005). As auxinas estimulam a divisão celular, além de apresentarem relações bastante importantes com ácidos nucléicos e proteínas, modificações da parede celular e estimulação de atividades enzimáticas (HARTMANN et al., 2002). Entre as principais funções biológicas das auxinas, podem-se citar o crescimento de órgãos, especialmente as raízes (HAISSIG, 1996).

O fato da enxertia tipo " $T$ " invertido não ter promovido resultados similares ao tipo " $\mathrm{T}$ " normal, pode estar relacionado ao corte horizontal ter sido realizado logo acima do nó da estaca, onde se localizava uma gema. Esse corte promoveu a indução da brotação da gema da estaca, desfavorecendo a translocação de água e reservas (carboidratos) para a borbulha enxertada, o que não ocorreu na enxertia tipo "T", pelo corte horizontal localizar acima da gema enxertada. Segundo Maia de Souza (2003), incisões horizontais acima de gemas caulinares em plantas de figueiras estimulam a saída da dormência das gemas e favorecem a emissão das brotações.

Apesar dos razoáveis resultados na enxertia de mesa por borbulhia em figueira, o percentual de brotação não foi satisfatório, o que pode ter sido causado pela insuficiente quantidade de reserva armazenada no fragmento enxertado, desfavorecendo a cicatrização da região enxertada. Já o baixo desenvolvimento da brotação pode ser atribuído a falta de nutrientes, pois as estacas permaneceram em areia durante a fase experimental.

\section{Conclusão}

Estacas de figueira a serem enxertadas devem ser tratadas com AIB;
O período de junho a agosto favorece o enraizamento das estacas e a porcentagem de sobrevivência de borbulhas;

A enxertia de mesa por borbulhia deve ser realizada pelo tipo " $T$ " normal.

\section{Referências}

ALVARENGA, A. A.; ABRAHÃO, E.; FRÁGUAS, J. C.; CARVALHO, V. L.; SILVA, R. A.; SANTA CECILIA, L .V. C.; CUNHA, R. L.; SILVA, V. J. Figo (Ficus carica L.). In: TRAZILBO JÚNIOR, J. P.; MADELAINE, V. (Org.). 101 Culturas - manual de tecnologias agrícolas. Belo Horizonte: EPAMIG, 2007. p. 365-372.

CONDIT, T. J. An interspecific hybrid in Ficus. Journal Hered, California, v. 6, p. 165-168, 1950.

DAVIES, F. T.; LAZARTE, J. E.; JOINER, J. N. Initiation and development of roots in juvenile and mature leaf bud cuttings of Ficus pumila L. American Journal of Botany, St. Louis, v. 69, n. 5, p. 804-811, 1982.

DUTRA, L. F.; KERSTEN, E. Efeito do substrato e da época de coleta dos ramos no enraizamento de estacas de ameixeira (Prumus salicina L.). Ciência Rural, Santa Maria, v. 26, n. 3, p. 361-366, 1996.

FACHINELLO, J. C.; HOFFMANN, A.; NACHTIGAL, J. C. Propagação de plantas frutiferas. Brasília: Embrapa, 2005. $221 \mathrm{p}$.

FOOD AND AGRICULTURE ORGANIZATION FAO. Fig. Disponível em: <http://faostat.fao.org>. Acesso em: 12 set. 2008.

HAISSIG, B. E. Metabolic process in adventitious rooting of cuttings. In: JACKSON, M. B. (Ed.). New root formation in plants and cuttings. Dordrecht: Matinus Nijhoff, 1996. p. 141-190.

HARTMANN, H. T.; KESTER, D. E.; DAVIES JUNIOR, F. T.; GENEVE, R. L. Plant propagation: principles and practices. 7. ed. New Jersey: Prentice Hall, 2002. 880 p.

INSTITUTO BRASILEIRO DE GEOGRAFIA E ESTATÍSTICA - IBGE. Produção agrícola municipal. Disponível em: <http://www.ibge.gov.br>. Acesso em: 12 set. 2008 .

MAIA de SOUZA, R. M. Fig culture techniques. Acta Horticulturae, Leuven, n. 605, p. 99-101, 2003. 
NORBERTO, P. M.; CHALFUN, N. N. J.; PASQUAL, M.; VEIGA, R. D.; PEREIRA, G. E.; MOTA, J. H. Efeito da época de estaquia e do AIB no enraizamento de estacas de figueira (Ficus carica L.). Ciência e Agrotecnologia, Lavras, v. 25, n. 3, p. 533-541, 2001.

PIO, R.; CHAGAS, E. A.; CAMPO DALL'ORTO, F. A.; BARBOSA, W. Manejo para o cultivo da figueira. Campo \& Negócio, Uberlândia, n. 27, p. 62-63, 2007.

PIO, R.; RAMOS, J. D.; CHALFUN, N. N. J.; GONTIJO, T. C. A.; MENDONÇA, V.; CARRIJO, E. P.; CHAGAS, E. A. Propagação de estacas apicais de figueira: diferentes ambientes, ácido indolbutírico e tipo de estaca. Ciência e Agrotecnologia, Lavras, v. 30, n. 5, p. 1021-1026, 2006.
POOLE, R. T.; CONOVER, C. A. Propagation of ornamental Ficus by cuttings. HortScience, Alexandria, v. 19 , n. 19 , p. 120-121, 1984.

RIBEIRO, I. J. A. Doenças da figueira. In: CORRÊA, L. S.; BOLIANI, A. C. (Ed.). In: SIMPÓSIO BRASILEIRO SOBRE A CULTURA DA FIGUEIRA, 1., 1999, Ilha Solteira. Anais... Ilha Solteira: UNESP, 1999. p. 151-164.

RIGITANO, O. A figueira cultivada no Estado de São Paulo. 1955. Tese (Doutorado em Agronomia) - Universidade de São Paulo. Escola Superior de Agricultura Luiz de Queiroz, Piracicaba.

TYLER, J. The root-knot nematode. California Agricultural Experimental States, Davis, v. 2, n. 1, p. 30, 1944. 
